\title{
Spatiotemporal Activation Time Estimation Improves Noninvasive Localization of Cardiac Electrical Activity
}

\author{
Matthijs JM Cluitmans ${ }^{1,2}$, Jaume Coll-Font ${ }^{3}$, Burak Erem ${ }^{4}$, Dana Brooks ${ }^{3}$, Pietro Bonizzi ${ }^{2}$, \\ Joël MH Karel ${ }^{2}$, Paul GA Volders ${ }^{1}$, Ralf LM Peeters ${ }^{2}$, Ronald L Westra ${ }^{2}$ \\ ${ }^{1}$ CARIM School for Cardiovascular Diseases, Maastricht University, The Netherlands \\ ${ }^{2}$ Department of Data Science and Knowledge Engineering, Maastricht University, The Netherlands \\ ${ }^{3}$ Department of Electrical \& Computer Engineering, Northeastern University, Boston, MA, USA \\ ${ }^{4}$ Harvard Medical School and Boston Childrens Hospital, Boston, MA, USA
}

\begin{abstract}
Electrocardiographic imaging (ECGI) reconstructs epicardial potentials and electrograms from body-surface electrocardiograms and a torso-heart geometry. For clinical purposes, local activation and recovery times are often more useful than epicardial electrograms. However, noise and fractionation can affect estimation of activation and recovery times from reconstructed electrograms. Here, we employ a method for activation and recovery time estimation that detects the simultaneous presence of spatial and temporal features associated with a passing wavefront and evaluate this in a series of canine experiments. We show that estimation of activation times is more accurate when this spatiotemporal approach is used, however, recovery times are best determined with a temporal-only approach. Additional spatial smoothing further benefits activation and recovery time estimation in all cases. This results in a median beat origin localization error of only one centimeter, which could expedite catheter-based diagnostic evaluation and ablation in clinical settings.
\end{abstract}

\section{Introduction}

Electrocardiographic imaging (ECGI) aims at noninvasively reconstructing the electrical activity on the heart, based on body-surface potential recordings and a patientspecific torso-heart geometry. [9] In the last decades, much progress has been made in ECGI and clinical applications occur with increasing frequency. [2] ECGI is based on the potential-based formulation of the inverse problem of electrocardiography, and reconstructs epicardial potentials and electrograms. For clinical purposes, local activation and recovery times are often more useful than epicardial electrograms. However, it can be difficult to estimate the activation/recovery times from electrograms. For example, the epicardial activation time is usually defined as the

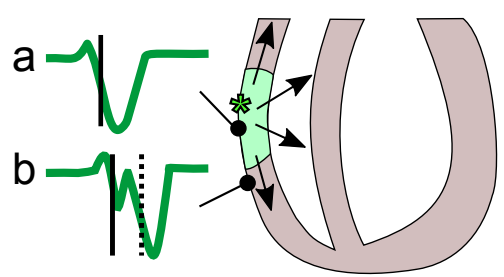

Figure 1. Illustration of the added value of spatial electrogram information in determining the moment of local activation. Electrogram $b$ is fractionated, with two deflections that could be selected by a temporal-only activation estimation method. Comparing this electrogram to neighboring electrogram $a$ makes the first deflection most likely the true activation time.

moment of steepest negative deflection in the electrogram. However, fractionation can be present in the electrogram, due to the activation pattern, noise, or the influence of illposedness. When fractionation is present, it can be difficult to determine which deflection is due to local activation. By incorporating information from neighboring electrograms, the most likely deflection can be chosen, as illustrated by Figure 1. This is exploited by a method proposed previously [5], which detects the simultaneous presence of the spatial and temporal features associated with a passing wavefront. In this study, we evaluate the accuracy of that spatiotemporal approach for estimating activation and recovery times and localization of beat origins in a series of canine experiments.

\section{Methods}

\subsection{In vivo recordings}

In vivo data were acquired in experiments with anesthetized dogs, which were approved by the institutional review committee for animal studies. In four normal anes- 


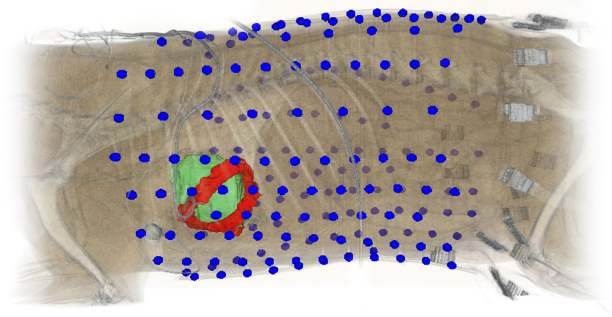

Figure 2. Validation data as collected in a canine experiment. Body-surface electrodes (blue), implanted electrodes (red) and the epicardial surface (green) were digitzied from a computated tomography (CT) scan.

thetized dogs, two silicone bands with 99 electrodes were implanted around the basal and mid-basal epicardium after thoracotomy [3]. After chest closure, body-surface electrodes (184 to 216, depending on torso size) were attached to the chest. This number of body-surface electrodes is more than sufficient to obtain a good reconstruction with traditional methods [1]. Unipolar potential recordings were obtained simultaneously by the epicardial and bodysurface electrodes. 93 beats were recorded during normal sinus rhythm or with pacing from the implanted electrodes.

\subsection{Inverse reconstruction of epicardial potentials}

The potential-based formulation of the forward/inverse problem of electrocardiography is based on the relation between potentials on a closed surface surrounding the heart, and the body surface [8]. The closed surface surrounding the heart is usually taken to be the epicardium. The forward problem can then be defined as:

$$
\Phi_{B}(t)=A \Phi_{H}(t)
$$

where $\Phi_{B}(t)$ are the potentials on the body surface at a specific time instant $t, \Phi_{H}(t)$ the potentials on the heart surface, and $A$ is the transfer matrix that relates these vectors. The transfer matrix captures the geometry and conductivity relation between the surfaces.

The goal of the inverse problem is to find the cardiac potentials $\Phi_{H}(t)$ from recorded body-surface potentials $\Phi_{B}(t)$ and a patient-specific transfer matrix $A$ (usually based on a computed tomography (CT) scan). However, the inverse problem is ill-posed, meaning that small variations in the body-surface potentials (e.g., due to noise) will result in disproportionately large changes in the computed cardiac potentials. Regularization, adding constraints to the solution, is needed to obtain a stable result. We applied the well-known zeroth-order Tikhonov method, penalizing the potential amplitude:

$$
\min _{\Phi_{H}(t)}\left\{\left\|A \Phi_{H}(t)-\Phi_{B}(t)\right\|_{2}^{2}+\lambda(t)\left\|\Phi_{H}(t)\right\|_{2}^{2}\right\}
$$

The regularization parameter $\lambda(t)$ balances the quality of fit with the amount of regularization. We determined this parameter with the L-curve method [7] and reconstructed epicardial potentials for all 93 recorded beats in the dogs.

\subsection{Estimation of activation and recovery times}

Activation and recovery times were determined from reconstructed electrograms with two different methods: a temporal-only method, and a spatiotemporal method.

The temporal-only approach defines the moment of activation as the moment of steepest voltage downslope $(\max -\partial \phi(t) / \partial t)$ during the QRS complex, where $\phi(t)$ is the potential at the epicardial node under consideration at time $t$. Recovery times were defined as the moment of maximum $\partial \phi(t) / \partial t$ during the $\mathrm{T}$ wave. These approaches were applied after smoothing the electrograms to reduce the influence of small deflections due to noise.

The spatiotemporal approach, proposed by Erem et al. [5], takes advantage of the spatial relationship between neighboring nodes and their potentials. They noted that not only the temporal signal (i.e., the local potential at a single node) changes quickly when an activation wavefront passes, but also the spatial gradient of potentials between neighboring nodes. More formally, for each epicardial node, they define the activation time $\tau$ as:

$$
\tau=\min _{t}\left\{\|D \phi(t)\|_{2} \cdot \frac{\partial \phi(t)}{\partial t}\right\}
$$

where $\phi(t)$ is the potential at the epicardial node under consideration at time $t, D \phi(t)$ is the approximated spatial gradient, and $\partial \phi(t) / \partial t$ the approximated temporal derivative.

Although designed for activation time estimation, we also applied this method to estimate recovery times; the minimization problem (equation 3 ) then becomes a maximization problem.

Ground truth activation and recovery times were determined from recorded electrograms with the temporal-only method.

\subsection{Additional spatiotemporal smoothing}

In addition, we explored the effects of the smoothing technique applied in [6]. This method smooths estimates of activation or recovery times over the heart surface. The final time $\tau_{D}$ is a balance between the current noisy estimation $\tau$ and a minimization of the Laplacian function on 


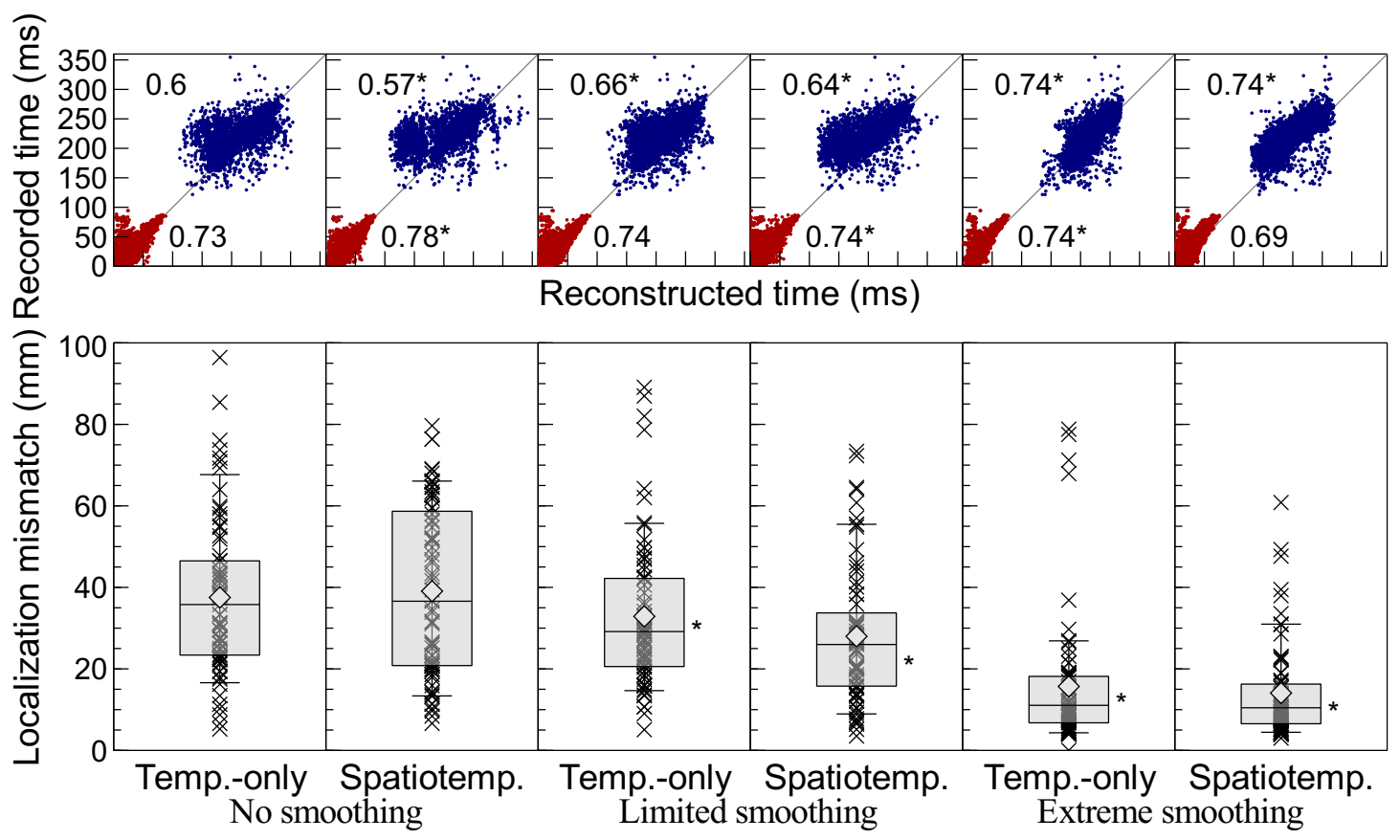

Figure 3. In vivo accuracy of the temporal-only ('Temp.-only') and spatiotemporal ('Spatiotemp.') approach to activation/recovery time estimation, with no (left two columns), limited (middle two columns), and extreme post-estimation spatial smoothing (right two columns). Top row: activation (red) and recovery times (blue) as determined from the recorded epicardial electrograms (vertical axis) and reconstructed epicardial electrograms (horizontal axis); R-values of the linear fit are shown. Bottom row: distance between known origin of pacing and reconstructed location of earliest activation. Box spans the $25-75 \%$ range; whiskers at $9-91 \%$ range; median indicated by horizontal line; mean by diamond. Asterisk $(*)$ indicates statistically significant difference from the temporal-only-no-smoothing results.

the surface. This trade-off is obtained through the following minimization:

$$
\min _{\tau_{D}}\left\{\left\|\tau-\tau_{D}\right\|_{2}^{2}+\gamma\left\|L \tau_{D}\right\|_{2}^{2}\right\}
$$

The first term, $\left\|\tau-\tau_{D}\right\|_{2}^{2}$, corresponds to a least squares minimization that impels the final estimates to be equal to the pre-smoothing estimation. The second term, $\left\|L \tau_{D}\right\|_{2}^{2}$, minimizes the surface Laplacian of the estimates. This is obtained through multiplication of $\tau_{D}$ by a numerical approximation of the Laplace operator $L$ obtained as in [4].

These two terms are balanced out with the parameter $\gamma$. For small values of $\gamma$, the final time estimates are similar to the noisy estimation, while for large values of $\gamma$ the resulting time estimates tend to zero. The choice of this smoothing parameter is determined by the user and depends on the final application. In this work, we explored the effect of different degrees of smoothing for estimation of activation and recovery times and beat localization. We analyzed the results without applying any smoothing $(\gamma=0)$, applying a limited $(\gamma=200)$ and an extreme amount of smoothing $(\gamma=200,000)$.

\subsection{Statistical evaluation}

For each epicardial electrode, linear correlation between recorded and reconstructed activation/recovery timings was assessed by means of Pearson's correlation coefficient R. Localization error was defined as the distance between the known location of pacing and reconstructed location of earliest activation. Results were statistically compared with Wilcoxon signed-rank tests (for paired measurements) or Wilcoxon rank-sum tests (for unpaired measurements), where the temporal-only-no-smoothing results were used for comparison.

\section{Results}

Figure 3 compares the temporal-only estimation of activation and recovery times with the spatiotemporal approach. It also investigates the added benefit of additional spatial smoothing.

Activation time estimation is most accurate when the spatiotemporal method is employed. Regardless of the estimation method, there is a no added benefit of additional spatial smoothing in terms of activation time accu- 
racy. However, the localization error does benefit significantly from more extreme smoothing.

Recovery time estimation accuracy is decreased when the spatiotemporal method, but benefits significantly from spatial smoothing.

Note that $\mathrm{R}$ values for activation/recovery times indicate correlation between the recorded and estimated times (all $p<0.05$ ); however, the asterisk (*) indicates not correlation, but distribution differences. This means that two results can have the same $\mathrm{R}$ value for similar linear fit, but with statistically different distributions.

\section{Discussion}

We have evaluated the accuracy of different methods to estimate activation and recovery times and pacing localization in ECGI. Activation time estimation is significantly improved when a spatiotemporal approach is used, compared to a temporal-only approach. This shows that incorporating spatial information is beneficial for estimation of activation times.

As a consequence of more accurate activation times, localization of beat origins (defined as the location of earliest activation) is improved as well. In this canine validation experiment, median accuracy of temporal-only beat localization was only $33 \mathrm{~mm}$. Spatiotemporal localization of beat origins had a similar median, but improved further to $26 \mathrm{~mm}$ with limited additional spatial smoothing, and 10 $\mathrm{mm}$ with extreme smoothing. Additionally, localization variability was reduced strikingly, especially with extreme additional spatial smoothing.

Interestingly, recovery time estimation did not improve with the spatiotemporal approach. This could be explained by the more gradual pattern of recovery, which results in less pronounced spatial gradients. Therefore, there is no added benefit of including a spatial gradient when estimating the upslope of the T wave, even the opposite: the spatiotemporal approach decreased accuracy of recovery time estimation. Regardless of the estimation method, there was added benefit from additional spatial smoothing, indicating that the use of spatial information is still relevant.

\section{Conclusion}

In conclusion, accuracy of activation times determined from reconstructed epicardial electrograms is improved when a spatiotemporal approach is used. Recovery times are best determined with a temporal-only approach. Additional spatial smoothing benefits recovery time estimation and the localization of beat origins. Combining the spatiotemporal approach to activation time estimation with additional spatial smoothing gives a median localization error of only one centimeter, which, upon translation, could expedite catheter-based diagnostic evaluation and ablation in clinical settings.

\section{References}

[1] Matthijs J M Cluitmans et al. "In-vivo evaluation of reduced-lead-systems in noninvasive reconstruction and localization of cardiac electrical activity". In: Computing in Cardiology. Vol. 42. 2015, pp. 2214.

[2] Matthijs J M. Cluitmans et al. "Noninvasive reconstruction of cardiac electrical activity: update on current methods, applications and challenges". In: Neth Heart J 23.6 (2015), pp. 301-11.

[3] Matthijs J M. Cluitmans et al. "Physiology-based Regularization Improves Noninvasive Reconstruction and Localization of Cardiac Electrical Activity”. In: Computing in Cardiology. Vol. 41. 2014, pp. 1-4.

[4] Burak Erem and Dana H. Brooks. "Differential geometric approximation of the gradient and Hessian on a triangulated manifold”. In: Proc IEEE Int Symp Biomed Imaging 2011 (2011), pp. 504-507.

[5] Burak Erem et al. "Spatiotemporal estimation of activation times of fractionated ECGs on complex heart surfaces". In: Conf Proc IEEE Eng Med Biol Soc 2011 (2011), pp. 5884-7.

[6] Burak Erem et al. "Using transmural regularization and dynamic modeling for noninvasive cardiac potential imaging of endocardial pacing with imprecise thoracic geometry". In: IEEE Trans Med Imaging 33.3 (2014), pp. 726-738.

[7] Per Christian Hansen and Dianne Prost O'Leary. "The use of the L-curve in the regularization of discrete ill-posed problems". In: SIAM Journal on Scientific Computing 14.6 (1993), pp. 1487-1503.

[8] Andrew J. Pullan et al. "The inverse problem of electrocardiography" In: Comprehensive Electrocardiology, edited by P. W. Macfarlane, A. van Oosterom, $O$. Pahlm, P. Kligfield, M. Janse, and J. Camm. Springer London, 2010, pp 299-344.

[9] C. Ramanathan et al. "Noninvasive electrocardiographic imaging for cardiac electrophysiology and arrhythmia". In: Nat Med 10.4 (2004), pp. 422-8.

Address for correspondence:

Matthijs JM Cluitmans, Department of Data Science and Knowledge Engineering, Maastricht University, P.O. Box 616, 6200MD Maastricht, The Netherlands, m.cluitmans@ maastrichtuniversity.nl 